\title{
Jeder für sich oder doch mehr für alle? Wege zu einer intensiveren regionalen Kooperation im Ruhrgebiet
}

\author{
Philipp Breidenbach • Rainer Kambeck • Florian Matz • \\ Christoph M. Schmidt
}

Eingegangen: 20. Januar 2012 / Angenommen: 18. Februar 2013 / Online publiziert: 30. März 2013

(C) Springer-Verlag Berlin Heidelberg 2013

\begin{abstract}
Zusammenfassung 53 Städte, darunter elf kreisfreie, vier Landkreise, drei Bezirksregierungen und zwei Landschaftsverbände - das Ruhrgebiet ist eine Region von wohl einzigartiger administrativer Fragmentierung. Gerade vor dem Hintergrund des steigenden Kostendrucks der öffentlichen Verwaltung steht diese Struktur im Zentrum der politökonomischen Diskussion. Die Vorschläge zur zukünftigen Entwicklung sind dabei nicht weniger vielschichtig. Sie reichen von einer Stärkung einzelner Städte als souveräne Zentren über die Schaffung einer Bezirksregierung Ruhr bis hin zur Verschmelzung zur Ruhrstadt. Auf der Basis theoretischer Kooperationskonzepte, einer sozioökonomischen Bestandsaufnahme und der Untersuchung einzelner bestehender Kooperationen im Ruhrgebiet wird in diesem Beitrag die Konzentration auf die Bildung einzelner funktionaler Kooperationen mit strikten Anforderungsprofilen und fallbezogenen Partnern vorgeschlagen. Die Anreize zur Überwindung des Status quo hin zu einer gelebten Kooperationskultur soll ein Wettbewerb mit monetären Gewinnen für vielversprechende und zukunftsgerichtete Kooperationen setzen.
\end{abstract}

Prof. Dr. C. M. Schmidt $(\bowtie) \cdot$ P. Breidenbach · Dr. R. Kambeck · F. Matz

Rheinisch-Westfälisches Institut für Wirtschaftsforschung (RWI),

Hohenzollernstraße 1-3, 45128 Essen, Deutschland

E-Mail: christoph.schmidt@rwi-essen.de

P. Breidenbach

E-Mail: philipp.breidenbach@rwi-essen.de

Dr. R. Kambeck

E-Mail: rainer.kambeck@rwi-essen.de

F. Matz

E-Mail: florian.matz@rwi-essen.de
Schlüsselwörter Ruhrgebiet · Regionale Analyse ·

Kooperative Handlungsoptionen · Regional Governance

Staying Alone or Reaching more Success Together? A Path for more Regional Cooperation in the Ruhrgebiet

Abstract Some 53 cities, 3 state governments and 2 Regional Associations - the Ruhrgebiet's unique administrative fragmentation has increasingly come under scrutiny, as cost pressure is mounting in public administration. Both in the political and the economic sphere, there are numerous proposals to overcome this situation. Some observers advocate the identification of some selected cities to be supported as regional centers, while other proposals even envisage one big "Ruhr-City". Based on theoretical cooperation concepts, a socio-economic review and the analyses of chosen cooperation projects, we suggest concentrating on cooperation along functional lines, initiated on a case-by-case basis. To overcome the status quo of reluctant cooperation, the right set of incentives will be needed. Specifically, we propose designing a competition offering potential cooperation partners substantial monetary benefits, if their proposal is picked as a winner.

Keywords Ruhrgebiet - Regional analysis - Cooperative strategies $\cdot$ Regional governance

\section{Einführung}

Nach Jahrzehnten des gelebten Strukturwandels zeigen die Daten der amtlichen Statistik und umfangreiche eigene Erhebungen (RWI 2011), dass das Ruhrgebiet eine wirtschaftlich nach wie vor sehr heterogene, polyzentrische 
Region ist. Seine Städte und Kreise stehen durch den absehbaren demographischen und den fortlaufenden strukturellen Wandel vor großen Herausforderungen. Doch die Integration der Verwaltungen in der Region hält mit diesem Wandel nicht Schritt, denn verfestigte Einzelinteressen bauen dafür nach wie vor nahezu unüberwindliche Hürden auf und entsprechende Initiativen verpuffen bislang ohne erkennbare Wirkung.

Dieser Beitrag zeigt, dass eine völlige Zusammenlegung administrativer Einheiten weder notwendig noch aktuell sinnvoll ist, um die Leistung der Verwaltung im Sinne der Bürger zu verbessern. Durch eine auf konkrete Aufgaben bezogene und möglicherweise zeitlich begrenzte Zusammenarbeit lassen sich bereits entsprechende Ressourcen einsparen und Synergien erzielen. Diese Einschätzung wird sowohl durch raumökonomische Überlegungen als auch durch die Analyse von im Ruhrgebiet derzeit bereits bestehenden Kooperationen gestützt.

Kooperationen werden in der ökonomischen Literatur intensiv als eine wichtige Option diskutiert, mit der in Situationen einer strategischen Interaktion - die Möglichkeiten und Resultate für jeden Akteur hängen vom Vorgehen der jeweils anderen Akteure ab - Zielkonflikte und Leistungsblockaden aufgelöst werden können (vgl. Fürst 2006: 37). Bei der Frage, wie staatliche Leistungen in effizienter Weise bereitgestellt werden können, sind Kooperationen jedoch grundsätzlich gegen die andere wichtige Option zur Organisation des Handelns in Situationen strategischer Interaktion, den Wettbewerb, abzuwägen. Der Wettbewerb zwischen administrativen Einheiten sollte dabei für solche Leistungen prägend sein, deren Auswirkungen nicht über die Grenzen der verantwortlichen Verwaltungseinheit ausstrahlen, sondern weitgehend nur dort für die Bürger direkt zu spüren sind.

Dieser Beitrag zeigt Wege auf, wie solche Kooperationen im Ruhrgebiet erfolgreich auf kommunaler Ebene umgesetzt werden können und welche Faktoren den Erfolg einer Kooperation beeinflussen. Zudem stellt der Artikel ein Gegenargument zu anderslautenden Forderungen, das Ruhrgebiet in eine „eigene ordnungspolitische Struktur mit demokratisch legitimierter Zuständigkeit" (Danielzyk/ Kirchhof/Knüpfer et al. 2010: 60) umzuwandeln, vor. Es wird hier insbesondere mit einem Kooperationswettbewerb ein zentrales Instrument vorgeschlagen, mit dem die Hemmnisse überwunden werden könnten, die bislang die stärkere Nutzung von Kooperationspotenzialen verhindern. Das Ziel einer effizienteren Verwaltungsstruktur könnte durch diese aufgedeckten Potenziale schneller erreicht werden.

Bevor im dritten Kapitel dieses Beitrags auf konkrete Kooperationen im Ruhrgebiet eingegangen wird, werden im zweiten Kapitel eine ausführliche Erörterung der theoretischen Grundlagen regionaler Kooperation und eine grobe Systematisierung der unterschiedlichen Kooperationsfor- men vorgelegt. Im vierten Kapitel werden Prinzipien und konkrete Vorschläge für künftige Kooperationsformen im Ruhrgebiet abgeleitet und im fünften Kapitel Handlungsempfehlungen dafür erarbeitet, wie der Weg in eine bessere (administrative) Zukunft der Region aktiv gestaltet werden kann.

\section{Regionale Kooperationen: Konzeptionelle Grundlagen}

\section{1 Ökonomische Ansätze}

Drei Ansätze regionaler Kooperationen werden in diesem Beitrag vorgestellt, der Metropolitan-, der „Public-Choice“und der „New-Regionalism“-Ansatz. Diese Ansätze stellen nicht die einzigen Theorien zur regionalen Kooperation dar, sie werden hier aber gewählt, weil sie exemplarisch für drei unterschiedliche Denkrichtungen stehen (Blume 2009: $42 \mathrm{ff}$.). Verweise auf einzelne Umsetzungsvorschläge werden bei den weiteren Ausführungen gegeben.

Der Metropolitan-Ansatz konzentriert sich darauf, mit den Skalenerträgen gemeinsamen Wirtschaftens und den auf Grund der gemeinsamen Aufgabenerfüllung eingesparten Transaktionskosten die Vorzüge einer umfassenden Kooperation auf allen Politikfeldern herauszuarbeiten. Ziel dieser Vorgehensweise ist es, die häufig zu beobachtende institutionelle Fragmentierung von Verdichtungsräumen zu überwinden (Kübler 2003: 536). Denn wenn jede Teilregion ihr Heil darin sucht, andere Teilregionen auszustechen, etwa bei umkämpften Prosperitätsquellen, wie der Ansiedlung eines großen Industrieunternehmens oder einer außeruniversitären Forschungseinrichtung, gerät oftmals das Wohl der Gesamtregion aus dem Blick. Größere (Verwaltungs-) Einheiten könnten nicht nur sinkende Durchschnittskosten, sondern möglicherweise aufgrund eines besser ausgebildeten oder stärker spezialisierten Personals eine Qualitätsverbesserung bei den angebotenen öffentlichen Leistungen erzielen. Bestimmte öffentliche Leistungen können sogar erst ab einer Mindestgröße der Gebietskörperschaft angeboten werden, wie beispielsweise eine leistungsfähige Verkehrsinfrastruktur.

Die Handlungsempfehlungen des Metropolitan-Ansatzes betonen insbesondere eine institutionell stark verankerte Kooperation der gesamten Region, bei der umfangreiche Befugnisse auf die entstehende größere Verwaltungseinheit übertragen werden. Notwendiger Bestandteil dieses Ansatzes sind folglich innerregionale Umlagesysteme, die finanzielle Belastungen zwischen Teilregionen ausgleichen. Zudem werden Formen von Bürgerbeteiligungen angestrebt, die sicherstellen, dass die größere Einheit weiterhin demokratisch legitimierte Entscheidungen trifft. Letztlich 
geht es beim Metropolitan-Ansatz um eine demokratisch legitimierte verwaltungstechnische Optimierung.

Derartige Überlegungen stehen auch hinter allen Vorschlägen zur Implementierung einer „Ruhrstadt“ in der Metropolregion Ruhrgebiet (vgl. z. B. Danielzyk/Kirchhof/Knüpfer et al. 2010). Beispiele von Gebietsreformen, die von solchen Ideen getragen werden, findet man in den Regionen Toronto, Montreal und Vancouver (Kübler 2003: 536). In Deutschland sind solche Kooperationen vor allem in homogenen Räumen, die klar von einem Zentrum dominiert werden, erfolgreich, z. B. in der Region Hannover oder der Städteregion Aachen (Benz/Fürst 2003: 198; Blume 2009: 143). Ein homogener Raum zeichnet sich dadurch aus, dass er wirtschaftlich eng zusammenhängt. So wirken sich gesamtwirtschaftliche Entwicklungen gleichmäßig in solchen Regionen aus. Die Homogenität geht oftmals mit der Monozentralität einer Region einher, ist aber nicht bedeutungsgleich. So kann das Ruhrgebiet der 1950er und 1960er Jahre - also in Zeiten der prosperierenden Montanindustrie - wohl als wirtschaftlich weitgehend homogen, sicherlich aber nicht als monozentrisch bezeichnet werden. Die Voraussetzungen für erfolgreiche Kooperationen im Sinne des Metropolitan-Ansatzes sind jedenfalls umso besser, je homogener eine Region strukturiert ist. Wenn alle Städte oder Teilregionen sehr ähnliche wirtschaftliche Profile aufweisen, haben diese wesentlich weniger Sorge um die angemessene Vertretung ihrer Interessen in der übergelagerten Regionalverwaltung.

Ist der Kooperationsraum in seiner wirtschaftlichen und sozialen Entwicklung hingegen heterogen und sind damit die Leistungsfähigkeit und die zu lösenden Aufgaben sehr verschieden, ist die gemeinsame Umsetzung von Verwaltungsprojekten schwierig und die Argumente des Metropolitan-Ansatzes verlieren an Überzeugungskraft. Heterogene Räume zeichnen sich dadurch aus, dass sie zum einen unterschiedlich stark von gesamtwirtschaftlichen Entwicklungen betroffen sind und zum anderen durch verschiedene Branchenorientierungen sehr unterschiedliche wirtschaftliche und soziale Entwicklungen durchleben. Stellt sich eine Region in einer Vielzahl wirtschaftlicher Kennzahlen als heterogen heraus, so ist eine einheitliche regionale Verwaltung in Anlehnung an den Metropolitan-Ansatz kaum zufriedenstellend für die einzelnen Teilnehmer realisierbar. Die Gefahr, dass eigene Probleme in einer regionsweiten politischen Debatte weniger stark antizipiert werden, ist in einer Metropolitan-Kooperation größer als in einer nichtkooperierenden Umgebung, in der jeder sein eigenes Wohl maximiert.

In der praktischen Umsetzung stoßen solche umfassenden Kooperationsbemühungen häufig auf starken Widerspruch der Bevölkerung, weil ein Verlust von Bürgernähe und Mitsprachemöglichkeiten sowie von regionaler Identifikation befürchtet wird. Die genannten Schwierigkeiten führten in einigen Fällen zu einer Ablehnung von Gebietsreformen. Beispiele dafür sind die Regionen Amsterdam, Rotterdam, der „Greater London Council“ sowie die Metropolregion Barcelona (Kübler 2003: 537). Im Ruhrgebiet selbst zeigt sich eine fortwährend bestehende Skepsis gegen von oben herab bestimmte Gebietsreformen. So wird noch heute jede Möglichkeit genutzt, die Ablehnung der Gebietsreformen der 1970er Jahre kundzutun, z. B. in der aktuellen Debatte um Kraftfahrzeugkennzeichen. ${ }^{1}$ Das Ruhrgebiet, als polyzentrische Region ohne dominierendes Zentrum, bietet dabei besonders ungünstige Voraussetzungen für umfassende Kooperationen - allein schon deshalb, weil eine Stadt fehlt, die als von allen anerkannter „LeadAkteur" fungieren könnte (Franz 2011: 389). Im Gegenteil: Weil mehrere Städte versuchen, einen Führungsanspruch zu erheben, herrscht eher eine „Kultur der Konkurrenz“ (Langhagen-Rohrbach/Fischer 2005: 76).

Beim „Public-Choice“-Ansatz (Tiebout 1956) stehen zunächst nicht der optimale Zuschnitt und die Finanzierung der Verwaltung im Blickpunkt des Interesses. Vielmehr wird die Frage gestellt, welche Formen der Interaktion staatlicher Einheiten, die in einer gegebenen räumlichen Abgrenzung ihre Leistungen erstellen, ein Höchstmaß an Nutzen für die Bürger der Region stiften können. Aus diesem Blickwinkel stellt sich heraus, dass dies häufig am besten durch die Schaffung von Marktstrukturen und die Intensivierung des Wettbewerbs erreicht werden kann. Eine gewisse administrative Fragmentierung ist aus dieser Sicht kein Problem, solange diese aus einer von den Bürgern gewünschten Differenzierung heraus entsteht. Vielfalt wird positiv bewertet und es wird gewürdigt, dass der Wettbewerb häufig die Qualität des Leistungsangebotes verbessert.

In einer modelltheoretischen Welt des „Public Choice“Ansatzes würden Städte, die ihr Angebot zu vergleichbar höherem Preis bereitstellen oder bei einem gegebenen Preis ein vergleichsweise geringes Angebot vorhalten, durch Abwanderung von Bürgern bestraft. Es kann nach den Erkenntnissen dieses regionalökonomischen Ansatzes also durchaus ein Ausdruck der Bürgerpräferenzen sein, wenn eine Stadt weniger Leistungen - etwa ein geringeres kulturelles Angebot oder weniger Freizeitmöglichkeiten - anbietet, aber dafür dem Bürger im Gegenzug auch geringere Steuer- und Gebührenzahlungen abverlangt.

Einzelne Kooperationen zwischen Städten und Gemeinden sind somit Ausdruck einer in diesem Fall effizienten Lösung zur Optimierung des öffentlichen Angebots. Administrative Kooperationen finden hier vor allem in besonders kapitalintensiven Bereichen statt, weil dort hohe Skaleneffekte erreicht werden. Da die Gemeinden von ihren Bürgern gedrängt werden, effizient zu wirtschaften, hätten sie - wenn die Vorstellungen des „Public-Choice“-Ansatzes zuträfen -

\footnotetext{
${ }^{1}$ Beispiele dafür sind Wattenscheid und Wanne-Eickel.
} 
einen großen Anreiz zur Umsetzung dieser gewinnbringenden Kooperationen (Kübler 2003: 538).

Die praktische Bedeutung des „Public-Choice“-Ansatzes steht und fällt aber mit der Mobilität der Bürger, mit der sie auf Leistungsunterschiede der Verwaltungseinheiten in den Regionen reagieren. Selbst in Kulturen mit einer hohen Mobilität führt nicht jede Ineffizienz der Verwaltung zu Abwanderungen und effiziente Strukturen lösen nicht automatisch Zuwanderung aus. Im Sinne des „Public-Choice“-Ansatzes darauf zu setzen, dass sich die zielführenden administrativen Kooperationen im Ruhrgebiet quasi von selbst, als Reflexion rationalen Bürgerwillens im regionalen Wettbewerb, ergeben würden, ohne dass es dazu weiterer Anstöße bedürfe, dürfte daher eine unzureichende regionalpolitische Strategie sein. Eine eher praxisbezogene Umsetzung des Public-Choice-Ansatzes diskutieren Frey und Eichenberger in dem Vorschlag zur Einrichtung von „Functional Overlapping Competing Jurisdictions (FOCJ)“ (Frey/Eichenberger 2001). Dieser Vorschlag ähnelt zwar sehr stark dem im Folgenden dargestellten Konzept des „New Regionalism“, notwendig ist aber hier eine „direkte demokratische Legitimierung und eine eigene Steuerhoheit der Körperschaft" (Blume 2009: 46). Eine Umsetzung dieses Vorschlags ist in Deutschland aber eher unrealistisch, weil es kaum Elemente der direkten Demokratie gibt und weil die Steuerhoheit auf der unteren Ebene nur schwach ausgeprägt ist. Auf der Ebene der Regionen ist die Steuerhoheit faktisch nicht gegeben.

Die beiden zuvor dargestellten Denkschulen drehen sich im Wesentlichen um die Diskussion der Frage, ob und unter welchen Bedingungen die institutionelle Fragmentierung in Verdichtungsräumen etwas Schlechtes oder Gutes ist. Die Realität zeigt jedoch, dass in den meisten Ballungsgebieten die politische Steuerungsleistung ohnehin durch ein Geflecht von miteinander vernetzten Kooperationsstrukturen zwischen den relevanten Akteuren stattfindet. Man erkennt darin zwar keine autonome Regierungsebene, wie vom Metropolitan-Ansatz gefordert, eine reine Zweckorientierung ohne die Relativierung der Gemeindeautonomie, wie sie im „Public-Choice“-Ansatz diskutiert wird, stellen diese Geflechte jedoch ebenfalls nicht dar (Kübler 2003: 539).

Mit diesem Blick auf die Realität wurde der „New Regionalism"-Ansatz hergeleitet (vgl. Blume 2009: 47 f.). Der Ansatz setzt an der Erkenntnis an, dass manchmal eine institutionalisierte und manchmal eine informelle Kooperation zielführend sind, und dass es oft nicht anzuraten ist, Wettbewerb zu hemmen. Nichtsdestoweniger setzt der „New Regionalism“-Ansatz auf das freiwillige Ausbauen von Kooperationsbeziehungen, die dabei aber als netzwerkartige Zusammenschlüsse organisiert sind. Diese Handlungsempfehlungen konzentrieren sich nicht nur auf die Zusammenarbeit der Gebietskörperschaften, also mehr oder weniger formell stabilisierte Akteurssysteme, sondern beziehen auch nicht-staatliche Akteure aus Wirtschaft, Wissenschaft und dem privaten Sektor mit ein.

Geeignet sind die netzwerkartigen und wechselnden Kooperationen nach dem „New Regionalism“-Ansatz vor allem für heterogene Räume, in denen für verschiedene Probleme unterschiedliche Kooperationspartner gesucht werden müssen. In der Praxis werden solche Bündnisse in Regionen genutzt, in denen Metropolitan-Ansätze nicht funktioniert haben, z. B. in der Region Rhein-Main (Blume 2009: 152 ff.). Diese pragmatische Vorgehensweise ist sinnvoll, weil nicht nur die konkrete Aufgabenstellung, sondern auch die jeweils lokal spezifischen Kombinationen von institutionellen, wirtschaftlichen, sozialen und politischen Faktoren die optimale Kooperationsform determinieren.

Ein großer Vorteil dieser flexiblen Form, Kooperation anzuregen und durchzusetzen, liegt in der Tatsache, dass die Konsolidierung einzelner Entscheidungsebenen - im Gegensatz zum Metropolitan-Ansatz - keine zwingende Voraussetzung für Kooperationen ist. Konsolidierung löst große Widerstände bei den betroffenen Instanzen aus und die Praxis zeigt, dass auch sinnvoll erscheinende Kooperationen daran scheitern. Regionalpolitische Strategien, die sich am Ansatz des „New Regionalism“ orientieren, diskutieren nicht diese Grundsatzfrage, sondern betonen die einzelnen Problemlagen und die für sie entwickelten kooperativen Lösungsansätze.

Die Kritik am „New Regionalism“-Ansatz stellt insbesondere auf die fehlende demokratische Legitimierung und die eventuell fehlende Transparenz für die betroffenen Bürger ab (Blume 2009: 48). Diese Kritik hat durchaus ihre Berechtigung: Der Grundgedanke des „New Regionalism"-Ansatzes ist, zur Lösung von Problemen Kooperationsarrangements zu bilden, die nicht an formal verfassten Institutionen festhalten. Die Bürgerbeteiligung findet aber typischerweise über eben diese formalen Institutionen statt, die in klar abgegrenzten Gebieten tätig sind. Somit folgt bei einer intensiven Umsetzung der Ideen des „New Regionalism" zwangsläufig eine Abkoppelung der demokratischen Einflussnahme von den Problemlösungsstrategien. Zudem ist der Ansatz anfällig für Einflüsse gut organisierter Interessengruppen.

Der „New Regionalism“-Ansatz stellt eine Abkehr von der Kooperationsfunktion der regionalen Steuerung hin zu einer Regional Governance mit flexiblen und wachsenden Strukturen dar (Fürst 2003: 442). Bei Kooperationen nach dem zuvor dargestellten Metropolitan-Ansatz liegt schon vorab der immer gleiche, große Kooperationsraum vor, auf dessen Ebene Aufgaben angegangen werden. Im „New Regionalism"-Ansatz liegen hingegen zunächst Aufgaben vor, für dessen Bewältigung die Akteure vor Ort mit ihren Initiatoren einen optimalen Kooperationsraum definieren. Es müssen also aus den bestehenden Strukturen her- 
aus kooperative Lösungen zur Effizienzverbesserung mit jeweils wechselnden Kooperationspartnern gefunden werden, was jeweils zu spezifischen Transaktionskosten (Suchkosten) führt. Daher gilt: Wenn die Möglichkeit vorliegt, trotz etwaiger Einführungswiderstände und trotz gegebenenfalls hoher Installationskosten eine Kooperation in Metropolitan-Form zu begründen, sollte diese genutzt werden. Allerdings gilt es grundsätzlich kritisch zu prüfen, ob die Voraussetzung einer hinreichenden Homogenität erfüllt ist.

In bestehenden institutionellen Strukturen zu arbeiten, bietet den Vorteil, keine Instanzen konsolidieren zu müssen. Der Nachteil besteht jedoch darin, dass alle Beteiligten, die für etwaige Ineffizienzen und Schieflagen - beispielsweise in den Bereichen Bildung, Kultur und Stadtplanung - im Status quo verantwortlich sind, weiterhin an den Entscheidungsprozessen teilnehmen. Damit dieser Status quo durch die Kooperation mehrerer Instanzen überwunden werden kann, müssen also entsprechende Anreize gesetzt werden. Diese Anreize können unterschiedlicher Natur sein, der offensichtlichste ist zweifelsohne in den meisten Bereichen der Kostendruck, also die Herausforderung, eine Aufgabe trotz eines beschränkten, gegebenenfalls sogar schrumpfenden Budgets erfüllen zu müssen.

Die beteiligten Institutionen werden aber nur dann die Erfahrung machen, dass ausschließlich eine Optimierung der Prozesse den auf ihnen lastenden Druck beseitigt oder zumindest mildert, wenn ihnen der vorhandene Kostendruck nicht durch eine übergeordnete Ebene abgenommen wird. Diese Einsicht wirft ein völlig anderes Licht auf (gut gemeinte) Rettungsaktionen für Kommunen, die meist ohne eigenem Zutun in eine finanzielle Schieflage geraten sind. Die Möglichkeit eines solchen „bail-out“ - also die Abwälzung von Lasten einer Gebietskörperschaft auf Dritte -, ohne dass sich die Akteure zu strukturellen Anpassungen verpflichten, muss glaubhaft ausgeschlossen werden. Die politische Situation ist allerdings eher durch solche BailOuts gekennzeichnet. ${ }^{2}$ Offenbar werden mit der Ankündigung, dass Kommunen die finanziellen Konsequenzen ihres Handelns gänzlich selbst tragen, erhebliche Stimmenverluste befürchtet. Aber trotz derartiger politökonomischer Hemmnisse bildet der „New Regionalism“-Ansatz aufgrund seiner Mischung aus analytischer Tiefe und pragmatischer Einschätzung der Durchsetzungsmöglichkeiten für administrative Verbesserungen ein belastbares Fundament

\footnotetext{
${ }^{2}$ Zumindest hinsichtlich der von den Kommunen eingeforderten Gegenleistung für den Erhalt von finanziellen Zuweisungen ist das in Nordrhein-Westfalen verabschiedete Stärkungspaktgesetz (Gesetz zur Unterstützung der kommunalen Haushaltskonsolidierung im Rahmen des Stärkungspakts Stadtfinanzen vom 9. Dezember 2011) eine Ausnahme. Denn von den Kommunen wird mit festen rechtlichen Vorgaben die Umsetzung von Haushaltssanierungsprogrammen gefordert. Die jährlich geplanten Hilfsleistungen fließen nur dann, wenn die Maßnahmen der Sanierungsprogramme erfolgreich umgesetzt werden (Stärkungspaktgesetz $§ 6$ ).
}

für Vorschläge zur administrativen Weiterentwicklung des Ruhrgebiets.

\subsection{Formen der regionalen Kooperation}

Die drei dargestellten Ansätze regionalökonomischen Denkens bilden den intellektuellen Unterbau, mit dem unterschiedliche Ausprägungen der regionalen Kooperation unterfüttert werden können. Dabei lassen sich im Grad der Institutionalisierung und im Aufgabenumfang in Anlehnung an Blume (2009: 53 ff.) jeweils drei Abstufungen der Kooperation unterscheiden: weiche Kooperationen, die nicht institutionalisiert sind, weiche Kooperationen, die institutionalisiert sind, und harte Kooperationen.

Weiche Kooperationen sind all jene Formen der regionalen Zusammenarbeit, die eher einen informellen und nicht verpflichtenden Charakter haben (vgl. Blume 2009: 56). Die Bandbreite reicht von Arbeitskreisen, die einem Erfahrungsaustausch dienen, über privat-rechtliche Organisationen, die ausgelagerte Funktionen der öffentlichen Hand übernehmen, bis hin zu Foren, in denen das Ziel regionaler Entwicklungskonzepte verfolgt wird. Weiche Kooperationen können den öffentlich-rechtlichen Institutionen beratend zur Seite stehen oder auf ihre Themen aufmerksam machen und somit zu effizienten Lösungen beitragen. Zusätzlich wird dadurch die Akzeptanz und Information zu politischen Prozessen und Entscheidungen in der Bevölkerung verbessert. Im Grad ihrer Verbindlichkeit lassen sich weiche Kooperationen nochmals unterscheiden: Nicht-institutionalisierte weiche Kooperationen bieten eine Offenheit, die vor allem für die Initiierung von Prozessen und für die Entwicklung von eher strategischen Konzepten hilfreich ist. Weiche Kooperationen kommen meist ohne eine große Ressourcenausstattung aus, für die Umsetzung von längerfristigen Zielen wird jedoch häufig eine stärkere Ressourcenausstattung benötigt. Kooperationen werden institutionalisiert, um anspruchsvolleren Anforderungen (z. B. ausgegliederte Aufgaben aus dem öffentlich-rechtlichen Bereich) besser entsprechen zu können.

Die harten Kooperationen zeichnen sich schließlich dadurch aus, dass sie in einem festen Rahmen stattfinden. Meist sind solche Kooperationen durch ein eng bindendes Vertragswerk fixiert und die Teilnehmer haben sich fest zur Mitarbeit verpflichtet. Verbindliche Kooperationen sind oftmals besser ausgestattet als weiche Kooperationen. Beispielsweise sind eigene Vertretungen (Büros) vorhanden sowie Mitarbeiter, die für die Durchführung der Kooperation zuständig sind. Während der Metropolitan-Ansatz allein auf harten Kooperationen fußt, kommen für „New Regionalism"-Kooperationen auch weiche Kooperationen in Frage. Falsch verstanden wäre der „New Regionalism"-Ansatz aber, würde er nur auf weiche Kooperationen 
beschränkt; harte Kooperationsformen sind ebenso möglich, je nach Eignung für die anstehende Aufgabe.

Harte, institutionalisierte weiche und nicht-institutionalisierte weiche Kooperationen lassen sich jeweils zusätzlich anhand ihres Aufgabenumfangs in funktional, multifunktional und ganzheitlich-integriert abgrenzen (vgl. Blume 2009: $59 \mathrm{ff}$.). ${ }^{3}$ Die funktionalen Kooperationen sind auf ein Aufgabengebiet spezialisiert und versuchen konzentriert auf diesen Bereich, bei der Problemlösung weitere Synergieeffekte zu nutzen. Häufig geschieht dies durch eine Beteiligung von Akteuren aus der Wirtschaft, wobei die öffentliche Hand oft selbst als Wirtschaftsakteur auftritt, etwa bei Stadtwerken, dem öffentlichen Nahverkehr oder der Entsorgung.

Hingegen übernehmen multifunktionale Kooperationen gleich mehrere Aufgabengebiete. Dies kann in verschiedenen Formen stattfinden. So sind sowohl der Regionalverband Ruhr (RVR), dem klar definierte Aufgaben der öffentlichen Hand übertragen wurden, als auch Verbundinitiativen, die sich den für sie relevanten Aufgaben zuwenden, multifunktionale Kooperationen. Dies führt dazu, dass das Gros der multifunktionalen Kooperationen breit gestreut ist. Abgrenzungsprobleme ergeben sich bei Kooperationen, die ihre Aufgabe zwar auf ein Thema begrenzen, dieses aber in großer Breite bearbeiten, wie z. B. die Gesellschaft „Ruhr2010“. ${ }^{4}$ Diese Kooperation kann als monofunktional (Thema Kultur) oder auch als multifunktional zur Jugendförderung, zum Freizeitangebot und zur Regionalwerbung gesehen werden. Eine eindeutige Zuordnung kann bei manchen Kooperationen (beispielsweise dem Regionalen Flächennutzungsplan oder der Universitätsallianz Metropole Ruhr ergänzen) nicht vorgenommen werden.

Die ganzheitlich-integrierten Kooperationen zeichnen sich dadurch aus, dass ihr Themengebiet nicht eingeschränkt ist. Die umfassendste Kooperation ist dabei der Zusammenschluss von Kommunen oder Kreisen. Hierbei werden alle Aufgaben auf die Kooperation übertragen. Kooperationen, denen fest definierte Teilaufgaben übergeben werden, wie z. B. der Regionalverband Ruhr, sind dagegen nicht ganzheitlich-integriert. Dabei ist es unerheblich, wie verbindlich eine Kooperation vereinbart ist, denn auch weiche Kooperationen, wie die „Initiative Ruhrstadt“" politischen Themen des Ruhrgebiets annehmen, zählen zu den ganzheitlich-integrierten Kooperationen. Kommen die Faktoren ,ganzheitlich-integriert“ und „hart“ zusammen, befindet man sich in Metropolitan-Kooperationen.

Viele ins Leben gerufene Kooperationen wollen sich allumfassend mit einer Problemstellung auseinandersetzen.

\footnotetext{
${ }^{3}$ Für weitere Konzepte für Kooperationsformen vgl. z. B. Healey (2002) und Fürst (2003).

${ }^{4}$ Vgl. http://www.essen-fuer-das-ruhrgebiet.ruhr2010.de/ (letzter Zugriff am 18.01.2013)

${ }^{5}$ Vgl. http://www.initiative-ruhrstadt.de (letzter Zugriff am 18.01.2013).
}

Eine Wirtschaftsförderungsgesellschaft in einem polyzentrischen Raum beispielsweise wird es sich meist zur Aufgabe machen, sowohl die Darstellung der Interessen der Region nach außen hin zu übernehmen als auch eine optimale Strategie für Ansiedlungen von Wirtschaftssubjekten zu entwickeln, also eine gewisse Steuerung nach innen anzustreben. Hierbei entsteht oft ein Spannungsverhältnis, denn eine solche Kooperation kann dem polyzentrischen Raum Vorteile bringen, die inter-kommunale Verteilung dieser Vorteile kann aber Schwierigkeiten aufwerfen (Blatter/Knieling 2009: 239 ff.). Gewichten potenzielle Teilnehmer einer solchen Kooperation später eventuell auftretende Probleme stärker als die erwarteten Vorteile, wird die Kooperation nicht zustande kommen. Eine klare Abgrenzung der Zuständigkeit der Kooperation kann dieses Problem lösen. Eine Kooperation könnte sich über externe Aufgaben definieren und ausschließlich darauf beschränkt sein, die Außendarstellung einer Region (in einem bestimmten Themenbereich) zu übernehmen. Der Folgeschritt - in diesem Fall die konkrete Ansiedlung - ließe sich dann unabhängig von der dabei verwirklichten Kooperation organisieren, etwa indem die lukrative Ansiedlung wettbewerblich entschieden wird.

\section{Das Ruhrgebiet}

\subsection{Sozioökonomische Bestandsaufnahme}

Bevor in diesem Kapitel eine Systematisierung der Kooperationslandschaft der Region vorgenommen wird, erfolgt zunächst ein Überblick über die wirtschaftliche Entwicklung des Ruhrgebiets in den vergangenen Jahren. Die zeitliche Entwicklung von Kennzahlen steht dabei im Fokus der Untersuchung. Häufig vorzufinden sind größere regionale Unterschiede in der kleinräumigen Betrachtung des Bruttoinlandprodukts (BIP) je Einwohner, die in der Regel durch die Verrechnung der Wertschöpfung am Arbeitsort zustande kommen. Wichtig für die Beurteilung der Homogenität respektive Heterogenität einer Region ist jedoch die zeitliche Veränderung der festgestellten Unterschiede. Vergrößern sich die Unterschiede im Zeitablauf, deutet dies auf unterschiedliche Potenziale in der langfristigen Entwicklung hin. Die festgestellten Unterschiede bei zentralen Indikatoren der wirtschaftlichen Leistungsfähigkeit (wie dem BIP je Einwohner oder den Bruttolöhnen) bestanden schon vor einem Jahrzehnt, sie sind aber seitdem tendenziell noch gewachsen. Dabei zeigt sich im Hinblick auf die Lage einzelner Städte und Kreise im internen Vergleich eine sehr hohe Persistenz: Die wirtschaftsstärkeren Städte und Kreise bleiben im Zeitablauf tendenziell vergleichsweise wirtschaftsstark, während diejenigen, die bereits vor einem Jahrzehnt im internen Vergleich zurücklagen, keinen Boden gutmachen konnten (RWI 2011: 17). 


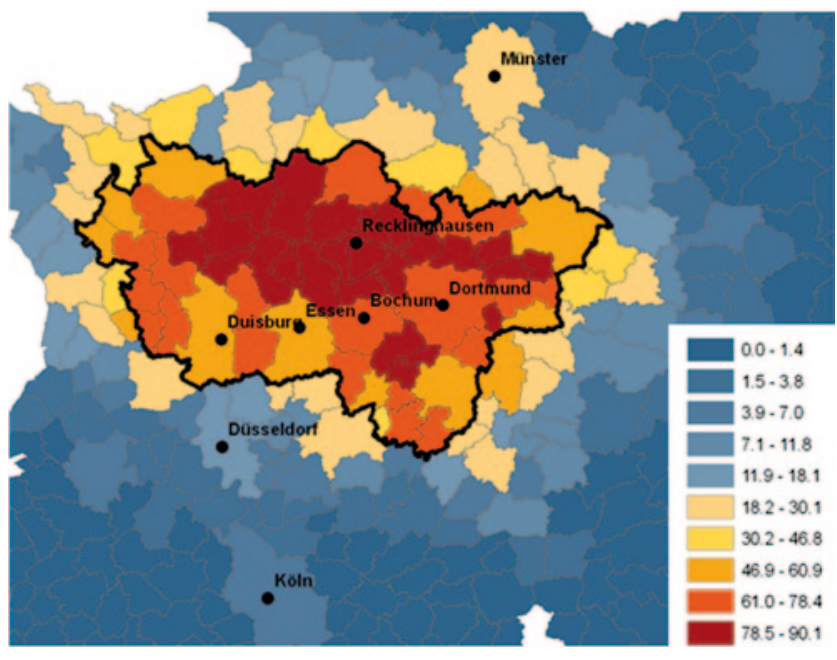

Abb. 1 Anteil der Auspendler in andere Gemeinden im Ruhrgebiet 2006 (in \% aller Auspendler). (Quelle: Eigene Darstellung nach Angaben der IT.NRW)

Der Arbeitsmarkt des Ruhrgebiets konnte insgesamt an der Erholung im Zuge der Hartz-Reformen partizipieren, die Arbeitslosenquoten variieren regional jedoch stark und entwickeln sich in den Städten und Kreisen zum Teil gegenläufig. Sie verstärken damit das Bild der heterogenen Entwicklungen im Ruhrgebiet (RWI 2011: 18 f.). Die Zahl der sozialversicherungspflichtig Beschäftigten stieg hingegen in den meisten Regionen des Ruhrgebiets, lediglich in den von Bergwerksstilllegungen betroffenen Gemeinden ist auch diese Entwicklung negativ, wodurch die Heterogenität der Region noch verstärkt wird. Insgesamt zeichnet sich jedenfalls anhand der wirtschaftlichen Kennzahlen keine einheitliche „Region Ruhrgebiet“" ab.

Die Pendlerströme führen ebenfalls nicht zur Herausbildung einer einheitlichen Gesamtregion (vgl. Abb. 1 und RWI 2011: 26). Der hier analysierte Anteil von Auspendlern, die in eine Ruhrgebietsstadt pendeln, zeigt eher eine Zweiteilung. Im nördlichen Ruhrgebiet wird in stärkerem Maße innerhalb der Ruhrgebietsgrenzen gependelt, im westlichen und südwestlichen Ruhrgebiet pendelt hingegen ein deutlich größerer Anteil der jeweiligen Auspendler in eine Stadt außerhalb des Ruhrgebiets. Das Ruhrgebiet stellt demnach keine in sich verflochtene, homogene Pendlerzone dar.

Neben den regionalen Wirtschaftsfaktoren zeigen auch die Bevölkerungsvorausberechnungen für die Region ein heterogenes Bild (RWI 2011: 31). Zwar wird für alle Städte im Ruhrgebiet eine negative Bevölkerungsentwicklung prognostiziert, der Bevölkerungsschwund variiert jedoch lokal sehr stark.

$\mathrm{Ob}$ und welche Kooperationen erfolgreich sind, hängt auch davon ab, wie stark sich die Bürger mit Regionen und Teilregionen identifizieren. Eine Befragung von 4.000 Bürgern des Ruhrgebiets und angrenzender Gemeinden ergab eine sehr hohe Verbundenheit mit dem Ruhrgebiet, wobei auch hier regionale Unterschiede vorliegen (RWI 2011: 49). Gerade die Aspekte, die die Bürger im Ruhrgebiet negativ wahrnehmen, bei denen also potenzieller Handlungsbedarf für die öffentliche Hand vorliegt, unterscheiden sich zwischen den Städten (RWI 2011: 47). ${ }^{6}$

\subsection{Bestehende Kooperationen und Kooperationspotenziale}

Im polyzentrischen Ruhrgebiet existiert bereits heute eine Reihe von Kooperationen, sowohl zwischen den Städten und Kreisen innerhalb des Ruhrgebiets als auch mit den Regionen außerhalb des Ruhrgebiets. Die Kooperationen sind sowohl öffentlich-rechtlich als auch von Seiten der Wirtschaft oder von Privatpersonen organisiert. Abbildung 2 ordnet ausgewählte Kooperationen nach der in Kapitel 2.2 vorgestellten Systematisierung. Eine umfassende Zusammenstellung aller Kooperationen hat das Institut für Landes- und Stadtentwicklungsforschung (ILS 2009) vorgelegt. Eine sehr anschauliche räumliche Darstellung von unterschiedlichen Kooperationsräumen im Ruhrgebiet findet sich bei Münter/Prossek (2011: 188 ff.). Im Folgenden werden einige Kooperationen genauer betrachtet, und nach Möglichkeit Aussagen über ihren Erfolg oder Misserfolg getroffen.?

Weil im Grunde alle Industrie-, Kultur- und Sportverbände funktionale Kooperationen darstellen, existiert eine Fülle solcher Kooperationen im Ruhrgebiet. Als funktionale Kooperationen sind aber auch solche zu bezeichnen, die in jüngerer Vergangenheit geschlossen wurden und die gemeinsam zentrale Leistungen der kommunalen Daseinsvorsorge anbieten, wie die „Kooperation Östliches Ruhrgebiet" (KÖR). Hier handelt es sich um eine weiche Kooperation der kommunalen Verkehrsunternehmen von Dortmund, Bochum, Gelsenkirchen, Herne und CastropRauxel sowie des Kreises Recklinghausen. Die KÖR wurde 1999 mit dem Ziel gegründet, Einkaufspolitik, Instandhaltung, Mitarbeiterschulung und Marketingmaßnahmen zu vereinheitlichen und den Austausch über technische Erfahrungen voranzutreiben. Ein rechtlicher Zusammenschluss zu einer juristischen Person ist nicht erfolgt. Diese räumliche Zusammenarbeit hat sich vor allem herausgebildet, weil die dort tätigen Verkehrsbetriebe bereit waren, einen Teil ihrer Entscheidungskompetenzen abzugeben.

Im Jahr 2010 wurde mit der VIA Verkehrsgesellschaft $\mathrm{mbH}$ eine ähnliche Kooperation von den Betreiberge-

\footnotetext{
${ }^{6}$ Eine ausführliche Darstellung der Methoden und der einzelnen Ergebnisse, die zu dem Schluss einer hohen Verbundenheit mit heterogenen Ausprägungen führen, finden sich in RWI (2011: 43 ff.).

${ }^{7}$ Informationen zu diesen Bewertungen wurden vielschichtig gesammelt. $\mathrm{Zu}$ Teilen beruhen sie auf direkten Gesprächen oder aus zusammengetragenen Informationen aus der Presse. In den Fällen, in denen sie auf offiziellen Quellen beruhen, werden diese angegeben.
} 


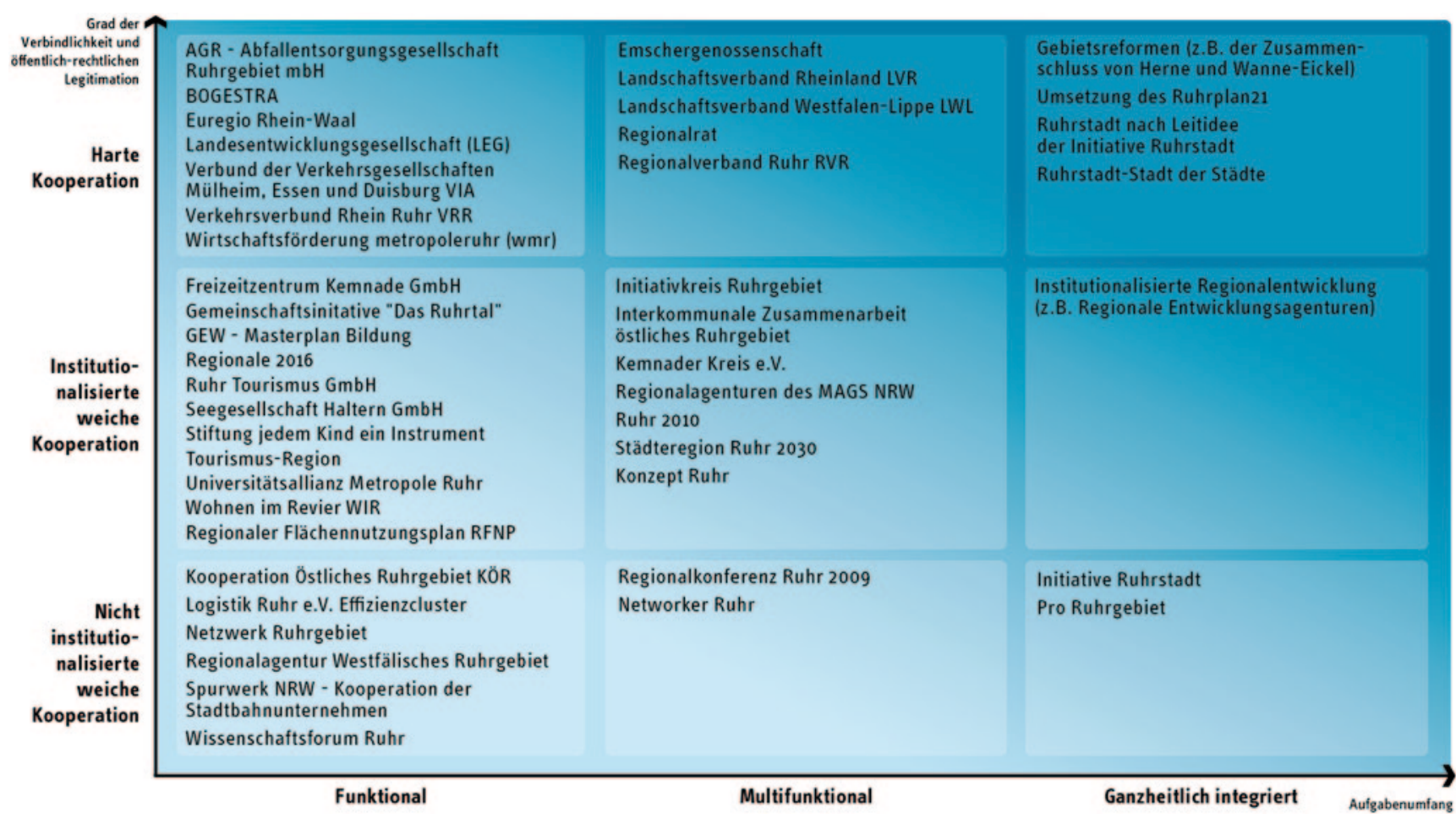

Abb. 2 Kooperationen im Ruhrgebiet. (Quelle: eigene Darstellung nach Blume 2009 und ILS 2009)

sellschaften der Städte Essen, Duisburg und Mülheim gegründet. Allerdings stellt die VIA eine wesentlich härtere Kooperation dar als die KÖR, weil mit der VIA ein Unternehmen geschaffen wurde, das als eigenständiger Betreiber fungiert, während bei der KÖR die kommunalen Betreiber bestehen bleiben. Auch in diesem Fall war - wie schon bei der KÖR - nicht die Homogenität der durch die Kooperation abgegrenzten Teilregion ausschlaggebend für die Gründung, sondern die bereits bestehende Vernetzung einiger Verkehrslinien. Wenngleich beim Stadtbahnverkehr diese bereits bestehenden Kooperationen als durchaus positive Beispiele genannt werden können, zeigt ein Vergleich der Netzpläne der Betreibergesellschaften, dass die interkommunale Verbindung zumeist nur in einer Hauptlinie zwischen den Hauptbahnhöfen besteht. Eine Verbindung von benachbarten Vororten verschiedener Städte, die nicht zufällig an dieser Hauptlinie liegen, ist nicht gegeben. Wie auch in anderen Analysen dargestellt, bietet der öffentliche Nahverkehr noch ein hohes Potenzial für weitere Kooperationen (Bogumil 2011: 583 f.).

Eine weitere funktionale, weiche Kooperation stellt die Universitätsallianz Metropole Ruhr (UAMR) dar - der Verbund der Universität Duisburg-Essen, der Ruhr-Universität Bochum und der Technischen Universität Dortmund. Diese Kooperation ist vertraglich fixiert und damit institutionalisiert, wobei aber die Souveränität der Hochschulen nicht eingeschränkt wird. Die Vereinbarung lässt vielmehr jede Form der Kooperation offen (Universitätsallianz Ruhr
2007: 3). Festgelegt wurde lediglich die Basis zur Zusammenarbeit, konkrete Projekte wurden nicht vereinbart. Weil die Universitäten der vier beteiligten Ruhrgebietsstädte angesichts der hohen Mobilität der Studenten innerhalb der Region gleichermaßen vor der Herausforderung stehen, immer umfangreichere Angebote zur „Spezialisierung“ des Studiums bereitzustellen, verbessert diese Kooperation die Qualität des Angebots und bietet zugleich ein hohes Potenzial zur Kostensenkung.

$\mathrm{Zu}$ den multifunktionalen Kooperationen zählen die beiden Landschaftsverbände Rheinland (LVR) und Westfalen-Lippe (LWL). Das östliche Ruhrgebiet gehört zum Landschaftsverband Westfalen-Lippe, Kreise und Städte westlich der Linie Bottrop, Gelsenkirchen, Bochum und Ennepe-Ruhr-Kreis zum Landschaftsverband Rheinland. Die Landschaftsverbände sind in der Kultur- und Denkmalpflege sowie in der Unterhaltung von Schulen und Krankenhäusern tätig. Die administrative Grenze der Landschaftsverbände wird kaum wahrgenommen. Die Unterscheidung zwischen Rheinland und Westfalen, wie sie durch die beiden Verbände vorgegeben wird, ist im Ruhrgebiet im Grunde überholt. Weil vom Regionalverband Ruhr (RVR) und den Bezirksregierungen ähnliche Aufgaben wahrgenommen werden, existieren nahezu zwangsläufig ineffiziente Überschneidungen von Kompetenzen.

Die bekannteste und größte Kooperation im Ruhrgebiet ist der erwähnte Regionalverband Ruhr (RVR). Der Verband wurde im Jahr 2004 gegründet. Durch seine Vorgänger, 
den Siedlungsverband Ruhrkohlenbezirk (SVR), gegründet 1920, und den Kommunalverband Ruhrgebiet (KVR), umbenannt 1978, blickt der Regionalverband Ruhr auf eine lange Historie zurück. Der RVR besteht als Pflichtverband für die 15 Kreise und kreisfreien Städte des Ruhrgebiets, dessen Aufgaben und Zwecke durch das Verbandsgesetz definiert sind. Zum Regionalverband Ruhr gehören 21 Tochtergesellschaften (RVR 2010: 5), deren Arbeit die im Regionalverband Ruhr gebündelten Kooperationen umsetzt. Im Gegensatz zu funktionalen Kooperationen, die sich von Aufgabe zu Aufgabe in immer wieder anderen räumlichen Konstellationen ergeben, umspannt die Aktivität der Tochtergesellschaften ein festes Gebiet.

Ein wichtiges Beispiel für eine solche Kooperation ist die Ruhr Tourismus GmbH (RTG), die das touristische Marketing der Region leitet und Tourismuskonzepte für das Ruhrgebiet erarbeitet. Hauptsächlich ist die RTG eine nach außen gerichtete Kooperation. Da der Faktor Tourismus starke Skaleneffekte aufweist, ist eine gemeinsame Marketinganstrengung auf regionaler Ebene überzeugend.

Eine weitere Tochter des Regionalverbandes Ruhr ist die Wirtschaftsförderung metropoleruhr GmbH (WMR) als ruhrgebietsweite Wirtschaftsförderungsgesellschaft. Ihre nach außen gerichteten Aufgabenfelder, wie die nationale und internationale Bewerbung des Standortes „Ruhrgebiet" durch gemeinsame Messeauftritte und gemeinsame Informationsbereitstellungen, sind tendenziell positiv zu bewerten. Es liegt jedoch auf der Hand, dass die nach innen gerichteten Aktionen wie die Vermittlung von Kontakten für potenzielle Investoren, nicht zufriedenstellend mit einer kooperativen Ausrichtung erfüllt werden können. Investoren, die sich für das Ruhrgebiet als Standort interessieren, werden von der WMR an die Städte vermittelt. Die Städte werben aber auch über andere, direkte Wege um potenzielle Investoren. Denn die Vertreter der kommunalen Wirtschaftsförderung haben durchaus einen hohen Anreiz, Investoren ohne Beteiligung der WMR zu akquirieren, weil sich die Stadt dann im Erfolgsfall nicht mehr mit „Konkurrenten“ auseinandersetzen muss.

Eine weitere nach innen gerichtete Aufgabe der WMR ist die koordinierende Unterstützung bei der Beantragung von EU-Fördermitteln, wie z. B. jüngst beim „Konzept Ruhr“. Ein ausschlaggebender Grund zur Schaffung der WMR war die Leitlinie der EU, Projekte bevorzugt zu behandeln, die in einer Kooperation von mehreren Kommunen eingebracht werden. Zur Umsetzung dieser multikommunalen Struktur wurde mit dem „Konzept Ruhr“ allerdings lediglich ein gemeinsamer Rahmen für viele individuelle Projekte geschaffen (WMR 2008). Diese Form der Umsetzung führt sogar im Vergleich zu Einzelanträgen zu einer Verschlechterung der Situation, weil es für Kommunen sinnvoll sein kann, möglichst viele eigene Projekte zu beantragen und somit eine für sie bessere Kompromisslösung auszuhandeln.
Spannend wäre es allerdings, in einer ausführlichen Evaluationsstudie genauer zu untersuchen, welche Zuständigkeiten aufgrund der Einrichtung der WMR von den einzelnen Wirtschaftsförderungsgesellschaften der Städte und Kreise tatsächlich an die WMR abgetreten werden. Weiterhin müsste analysiert werden, welche konkreten Einsparungen bei diesen Gesellschaften im Zuge dieser Wandlungen vorgenommen wurden und welche Doppelanstrengungen in der Praxis noch vermieden werden könnten. Es liegt allerdings nahe, dass sich die WMR wohl darauf konzentrieren sollte, ihre nach außen gerichtete Kooperation zu stärken und ihre Potenziale und Mittel auf die Erfüllung dieser Aufgabe auszurichten.

Der Regionalverband Ruhr übernimmt zudem Aufgaben in der Kulturförderung und als Betreiber von Freizeitflächen, etwa die Unterhaltung der Revierparks. Politische Positionen und Strategien des Verbands werden durch die Verbandsversammlung festgelegt. Dieses „Ruhrparlament“ setzt sich aus den Mehrheitsverhältnissen in den Stadt- und Kreisräten der RVR-Mitglieder zusammen. Seit den Kommunalwahlen in Nordrhein-Westfalen 2009 liegt auch die Aufgabe der Regionalplanung und der Erstellung von Flächennutzungsplänen bei der Verbandsversammlung (Bogumil 2011: 585). Die zuständige Regionalplanungsbehörde ist der Regionalverband Ruhr (RVR) selbst, er berät die Verbandsversammlung bei den Themen Städtebau, Wohnungsbau, Schul- und Sportstättenbau, Krankenhausbau, Verkehrs-, Freizeit- und Erholungswesen, Landschaftspflege, Wasserwirtschaft, Abfallbeseitigung, Kultur und Tourismus. $^{8}$

Der Regionalverband Ruhr ist nach der zuvor beschriebenen Systematik eine harte Kooperation mit multifunktionalem Aufgabenbereich. Überlappende Kompetenzverteilungen auf verschiedenen Verwaltungsebenen sind dabei oftmals ein Hindernis für eine effektive Aufgabenerfüllung: Zum einen sind Zuständigkeiten häufig „vertikal“ zwischen Stadt oder Kreis und dem RVR nicht klar verteilt, weil die Städte oftmals daran zweifeln, ob ihre Ziele auch angemessen vom RVR verfolgt werden. Deshalb behalten sie sich zum Beispiel mit Teilkompetenzen „Vetorechte" vor, um bei Planungen des Regionalverbandes nicht benachteiligt zu werden. Zum anderen gibt es ,horizontale" Kompetenzüberlappungen, zum Beispiel zwischen Landschaftsverbänden und dem Regionalverband Ruhr, die beide Kultur- und Freizeiteinrichtungen betreuen und unterstützen.

Für eine effektive und zielgerichtete Regionalpolitik durch den Regionalverband Ruhr müssten mehr Kompe-

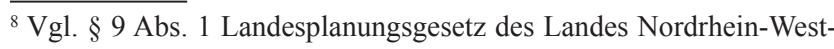
falen.
} 
tenzen vollständig in dessen Verantwortung liegen. ${ }^{9}$ Dazu müssten die Kreise und die kreisfreien Städte ihre Befugnisse konsequent auf den Regionalverband Ruhr übertragen. Dies wäre aber gleichbedeutend mit der Abgabe von Machtbefugnissen auf der Ebene der Kreise und der kreisfreien Städte. Vor allem Letztere haben ein großes Interesse daran, ihre Entscheidungshoheiten nicht vollständig aufzugeben (vgl. Blume 2009: 142 f. mit Beispielen für den Raum Hannover). Da gerade diese Verwaltungsebene das Ruhrparlament des RVR stellt und damit die Leitlinien des Regionalverbandes prägt, ist kaum zu erwarten, dass der Regionalverband Ruhr zukünftig eine machtvollere Position beziehen wird (Laurin 2010).

Einige Mitglieder des Regionalverbandes Ruhr wollen eine stärkere Unabhängigkeit und eine führende Rolle in Teilregionen erlangen, um eigene Interessen gezielter verfolgen zu können (vgl. Blotevogel 2001: 21). Auf Initiative einiger Bürgermeister wurde deshalb als Gegenentwurf zum Regionalverband Ruhr im Jahr 2008 der „Städtebund Ruhr" gegründet, der mit flexiblen Kooperationsformen ein Pendant zum Regionalverband Ruhr darstellen sollte. Dieses Projekt wurde allerdings nicht umgesetzt. Hingegen hat sich die „Städteregion Ruhr 2030“, eine Kooperation aller kreisfreien Städte des Ruhrgebiets mit ähnlich flexiblen Ausgestaltungsformen, etabliert. Sie stellt eine Plattform für Kooperationsprojekte dar und kann somit als weiche, eher multifunktionale Kooperation bezeichnet werden. Die auf dieser Plattform entstehenden Projekte können in ihrer Verbindlichkeit allerdings härter sein und sich monofunktional eine konkrete Aufgabe wie zum Beispiel die regionale Flächennutzungsplanung vornehmen. In diesem Projekt haben sich sechs Städte zusammengeschlossen und die durch die Landesregierung gegebene Möglichkeit zur Erstellung eines Regionalen Flächennutzungsplanes umgesetzt.

Die Forderungen der „Initiative Ruhrstadt“ formulieren derzeit die prominentesten Positionen für eine ganzheitlichintegrierte harte Kooperation im Ruhrgebiet. Auch wenn die aktuelle Aktivität dieser Initiative lediglich weiche Kooperationen beinhaltet, würde die Umsetzung ihrer Kernforderung das Verwaltungsgefüge weitreichend verändern. Nach den Vorstellungen der Initiative sollen wesentliche Kompetenzen der einzelnen Städte und Gemeinden auf ein neu zu wählendes „RuhrStadtparlament“ und einen „Ersten Bürgermeister" übergehen (Danielzyk/Kirchhof/Knüpfer et al. 2010: 18 und 49). Ein Kernproblem dieses Ansatzes besteht aber - wie auch beim Regionalverband Ruhr - in der mangelnden Anreizverträglichkeit, denn es fehlt an einer über-

\footnotetext{
${ }^{9}$ Ein positives Beispiel für eine solche Kompetenzübertragung ist die beschriebene Übergabe der Regionalplanungskompetenz von den Bezirksregierungen an den Regionalverband Ruhr. Die hier angesprochene Problematik bezieht sich vor allem auf die Kompetenzübergabe von der unteren Ebene (Städte und Kreise) an den Regionalverband Ruhr.
}

zeugenden Motivation für die politisch Verantwortlichen in den Kommunen, wesentliche Teile ihrer Zuständigkeiten auf eine höher angesiedelte Ebene zu übertragen. Die Gründung und Akzeptanz der oben beschriebenen ,Städteregion Ruhr 2030“ als Alternative spricht jedenfalls gegen den Willen der kreisfreien Städte zur Einführung einer Ruhrstadt.

Wenngleich die in diesem Kapitel angesprochenen Kooperationen bereits ein breites Themenspektrum der öffentlichen Daseinsvorsorge abdecken, bleiben weitere Themen, bei denen Kooperationen oder eine stärkere Zusammenarbeit sinnvoll sind, offen. Zudem werden in den genannten Bereichen die Potenziale bei weitem noch nicht ausgeschöpft. Ein Beispiel hierfür ist das Schulwesen. Mittelfristig werden die heute teils schon aktuellen Entwicklungsplanungen im Schulwesen flächendeckend alle Städte und Gemeinden im Ruhrgebiet betreffen. Effiziente Lösungsansätze sollten sich dabei nicht an den Stadtgrenzen orientieren. Kooperationen stellen hier zwar teilweise schon lange gelebte Praxis dar, von einer flächendeckenden Zusammenarbeit kann jedoch noch keine Rede sein. Gerade im Schulwesen könnten Kooperationen dazu beitragen, den Herausforderungen aufgrund der steigenden Nachfrage nach ganztägiger Betreuung sowie des demographischen Wandels zu begegnen.

Mit der alternden Bevölkerung ergeben sich weitere Herausforderungen. Dies betrifft nicht nur die Errichtung und Unterhaltung von Alten- und Pflegeheimen, sondern auch den gesamten Themenkomplex ,altengerechtes Wohnen und Leben". Weil dabei die Investitionen privater Unternehmen unverzichtbar sein werden, würden sich netzwerkartige Kooperationen nach den Vorgaben des „New Regionalism“ im besonderen Maße eignen.

Bei weitem noch nicht erschöpft sind im Ruhrgebiet die Möglichkeiten kooperativer Projekte zur Flächennutzung. Es gibt zwar positive Beispiele wie im Fall der Städte Gelsenkirchen und Herten, die gemeinsam die Folgenutzung des ehemaligen Zechengeländes Westerholt bestreiten, aber gerade bei den ehemaligen Zechengeländen bestehen noch erhebliche Kooperationspotenziale. Auch im Bereich der städtischen Freizeit- und Sportanlagen, vor allem bei zahllosen Fußballplätzen sowie Frei- und Hallenbädern, sind weitere spezifische Kooperationen sinnvoll, weil das Angebot interkommunal wesentlich besser aufeinander abgestimmt und genutzt werden kann. Ebenso bieten große Sport- und Freizeitanlagen, wie Eislaufhallen oder Freizeitbäder, Potenziale für kooperative Handlungsmöglichkeiten, vor allen den Unterhalt solcher Anlagen betreffend (Speer/ Denkel/Winterhager et al. 2009: 92). Weitere Beispiele und vertiefende Analysen von bestehenden Kooperationen finden sich zum Beispiel in Blotevogel (2009), ILS (2009), Reicher/Kunzmann/Polívka et al. (2011) und RWI (2011). 


\section{Indikatoren erfolgversprechender Kooperationen}

Analysen der Kooperationslandschaft zeigen, dass im Ruhrgebiet vor allem solche Kooperationen existieren, die nicht von administrativer Seite geformt sind (Münter/Prossek 2011: 192 ff.). Die Fülle an von unten entstandenen Initiativen zeigt, dass ein großer Bedarf nach solchen Projekten in der Region vorliegt. Wie im dritten Kapitel dargestellt, gibt es durchaus bereits Kooperationen im Ruhrgebiet, die sich durch sinnvolle und gut organisierte Zusammenarbeit auszeichnen. Dies ist bei weitem nicht der Regelfall. Zudem werden die Chancen, die sich daraus ergeben würden, die Replizierbarkeit solcher positiver Beispiele positiv herauszustellen, bisher kaum genutzt. Oftmals werden Projekte ad hoc und ohne klare Leitlinien angestoßen. In den folgenden Ausführungen werden deshalb grundsätzliche Anforderungen an Kooperationen ausgearbeitet, die eine erfolgreiche Umsetzung von Kooperationsprojekten deutlich verbessern. Diese beruhen zum Teil auf Arbeiten zum Regionalmanagement (vgl. z. B. Knieling 2003: 467), zum Teil werden sie auf der Basis ökonomischer Zusammenhänge vorgenommen - Letzteres in erster Linie mit Bezug auf den „New Regionalism“-Ansatz.

Damit Kooperationen zu einer besseren administrativen Zukunft der Region führen können, sollten sie folgende zentrale Prinzipien erfüllen. Kooperationen müssen den Akteuren klar definierbare Vorteile bieten, sonst werden sie sich nicht dafür engagieren. Steht die spezielle Problemlösung im Vordergrund und nicht der Versuch einer ruhrgebietsweiten Aufgabenerfüllung, bestehen positive Anreize für die betreffenden Kommunen, mit anderen gemeinsam an Problemlösungen zu arbeiten. Grundsätzlich sollte in einer heterogenen Region für jede kooperative Initiative das Problem selbst im Vordergrund stehen und dadurch der optimale Teilraum zur Lösung gefunden werden. Es sollten also Kooperationen nach funktionalen anstatt nach territorialen Gesichtspunkten gewählt werden (Fürst 2003: 442). Somit kann das gesamte Ruhrgebiet zur Erreichung bestimmter Ziele durchaus ein optimaler Kooperationsraum sein. Die Einbeziehung des gesamten Ruhrgebiets sollte aber nicht die Grundvoraussetzung für jegliche Kooperation sein. Im Gegenteil: Für viele Aufgaben dürften Kooperationen in Teilräumen oder über das Ruhrgebiet hinausgehend einen passenderen Rahmen darstellen (Bogumil 2011: 589).

Die Teilnahme an einer Kooperation sollte freiwillig sein, denn dies garantiert, dass in den Kooperationen nur diejenigen regionalen Akteure zusammenarbeiten, die sich davon echte Vorteile bei der Lösung anstehender Probleme versprechen. Kooperationen sollten ein konkretes, klar definiertes Ziel haben und von Anfang an einem konkreten Zeitplan folgen. Der Umfang einer Kooperation sollte verbindlich festgelegt werden, ebenso wie die Kompetenzen und Verantwortlichkeiten.
Insbesondere bei Überschneidungen mit der kommunalen Verwaltung ist klar festzulegen, welche Kompetenzen jeweils an die Kooperation übertragen werden. Unklarheiten über die tatsächlichen Kompetenzen einer Kooperation führen zu unnötigen Unsicherheiten über die Umsetzung gefundener Lösungen. Zudem sollte die Aufteilung der anfänglichen Kosten und der erwarteten Erträge einer Kooperation auf die einzelnen Mitglieder bereits im Vorhinein geklärt werden. Insbesondere eine zu spät vorgenommene Zuordnung von erzielten ,Gewinnen“ führt oftmals zu Verteilungskonflikten und gefährdet den Fortbestand einer Kooperation.

Ziele, Aufgaben und Kompetenzen von Kooperationen sollten transparent dargelegt werden, damit die Resultate auch effektiv bewertet werden können. Um die Transparenz möglichst umfassend zu gestalten, sollte bereits zum Start einer Kooperation eine Evaluation vereinbart werden. Für die Kooperationspartner selbst ist dies wichtig, weil sie ihre eigene Strategie überprüfen und gegebenenfalls notwendige Korrekturen vornehmen können. Gleichwohl kann eine Evaluierung mit guten Ergebnissen aber auch die eigene Position gegenüber Kritikern stärken. Nach außen können positive Bewertungen zudem die Attraktivität kooperativen Handelns erhöhen und weitere Kooperationspartner anlocken. Mithilfe hoher Transparenz kann der Kritik der mangelnden Klarheit über den Einfluss privatwirtschaftlicher Akteure entgegnet werden. Die demokratische Legitimierung von „New Regionalism“-Kooperationen wird allein dadurch zwar nicht erreicht, ein zu hoher Einfluss mächtiger Akteure durch intransparente Strukturen kann so aber verhindert werden. Insgesamt gewinnt die Beteiligung privater Akteure angesichts der in vielen Kommunen (gerade des Ruhrgebiets) prekären Haushaltslage weiter an Bedeutung.

\section{Gestalten durch richtig gesetzte Anreize}

Um einen Übergang aus der administrativen Verfassung des Ruhrgebiets im Status quo in die wünschenswerte Position einer auf dem „New Regionalism“-Ansatz basierenden Kooperationskultur zu schaffen, soll - der Vorgehensweise der Wohlfahrtsökonomik folgend - ein hypothetisches Gedankenexperiment aus der übergreifenden Sicht eines Planers genutzt werden. Damit kann eine gesellschaftliche Problemlage zunächst in ,abstrakter“ Form diskutiert werden. Welche Anstrengungen sollten unternommen werden, um einen verbesserten administrativen Zuschnitt in der Region zu erreichen oder ersatzweise über Kooperationen Ressourcen zu sparen und Synergieeffekte zu heben? In der Praxis könnten die Ergebnisse dieser abstrakten Überlegungen als Richtschnur für die regionalpolitische Strategie der Landesregierung Nordrhein-Westfalens, aber auch für Institutionen, die explizit der Förderung des Wohls der Region 
gewidmet sind, dienen. Einem gemeinwohlorientierten Akteur bieten sich im Prinzip drei Möglichkeiten, mit sehr unterschiedlichen Erfolgsaussichten.

Er könnte erstens die geeignete Form der kommunalen Kooperationen selbst identifizieren und je nach den eigenen Möglichkeiten durchsetzen. Ein solcher Ansatz wäre recht aussichtslos, weil die Städte und Kreise sich aufgrund ihres Selbstbestimmungsrechtes jede ihnen missliebige Weichenstellung blockieren können. Darüber hinaus stellt sich die Frage, wie es die übergeordnete Stelle schaffen soll, die genauen Vor- und Nachteile bestimmter Ausprägungen einer möglichen Kooperation trennscharf zu erkennen, denn hier haben die potenziellen Partner grundsätzlich einen erheblichen Informationsvorsprung. Zudem ist es nicht realistisch, dass die Landesregierung oder am Gemeinwohl orientierte Ruhrgebiets-Institutionen ihre Vorstellungen quasi per Weisung durchsetzen können, so vernünftig und zielführend diese im Einzelfall auch sein mögen.

Als zweite, ebenfalls letztlich nicht sehr vielversprechende Option böte sich für den Planer an, Vorschläge für mögliche Kooperationsprojekte zu erarbeiten und für deren Umsetzung bei den betroffenen Städten und Kreisen zu werben. Dies hat jedoch angesichts der vorhandenen Beharrungskräfte zur Beibehaltung einer unantastbaren kommunalen Autonomie ebenfalls geringe Erfolgsaussichten. Denn zum einen bestehen die oben erwähnten Informationsvorsprünge seitens der einzelnen Städte und Kreise und der dort verantwortlichen Politik weiter. Zum anderen kann man sich leicht vorstellen, dass selbst prinzipiell zielführende Kooperationsvorschläge aus strategischen Gründen eigentlich immer als in der Praxis undurchführbar dargestellt werden können, wenn sie bei den potenziellen Partnern nicht gewollt sind. Eine umfassende Veränderung der Kooperationskultur und eine Beschleunigung des Wandels im Ruhrgebiet werden aus gut gemeinten Appellen und Ratschlägen heraus nicht zu bewirken sein.

Die dritte und eindeutig vorzuziehende Option bestünde

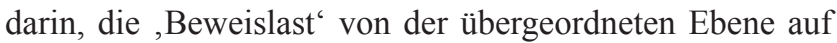
die einzelnen Akteure in den Städten und Kreisen zu verschieben. Die Akteure selbst sollten konkrete Kooperationspotenziale identifizieren und ihre Umsetzung planerisch vorbereiten. Dann wären ihre Informationsvorteile ideal kanalisiert und es bestünde zumindest die Hoffnung, dass die zielführendsten Kooperationsprojekte zuerst verwirklicht werden und andere Aktionen unterblieben. Weil die Notwendigkeit und die Vorteilhaftigkeit von kooperativem Handeln am besten auf der direkt involvierten Politikebene erkannt werden, ist es zwingend notwendig, dass Bestrebungen zum gemeinsamen Handeln hauptsächlich auf dieser unteren politischen Ebene initiiert werden (bottom-up). Der Umsetzungswille bei selbst entworfenen Kooperationsideen dürfte wesentlich größer sein.
Um einen Prozess solcher selbstbestimmter Kooperationen $\mathrm{zu}$ intensivieren, müssen allerdings die richtigen Anreize gesetzt werden, diese können negativer oder positiver Natur sein. Als negative Anreize kommen vor allem die Kürzung finanzieller Zuweisungen der Landesregierung an die Kommunen in Betracht, die gerade in den aktuellen Zeiten der großen Haushaltsnöte einen erheblichen Hebel darstellen dürften, um die Städte und Kreise als Gegenleistung für einen angestrebten Bail-out anzuhalten, ihre Bemühungen um effizienteren Ressourceneinsatz zu intensivieren. Nichts anderes als diese Strategie verfolgt der „Internationale Währungsfonds“ (IWF), wenn er als Gegenleistung für Notkredite an klamme Staaten strukturelle Anpassungsprogramme durchsetzt, um damit deren langfristige Solvenz zu sichern. ${ }^{10}$ Um diesen Hebel zum Wohle der Bürger der Region einzusetzen, bedarf es seitens der Landesregierung natürlich erheblicher politischer Standfestigkeit.

Komplementär dazu lassen sich positive Anreize setzen, die ebenfalls die Eigeninitiative der potenziellen Kooperationspartner anregen könnten. Ein Blick auf die Strukturpolitik zeigt, dass Wettbewerbssysteme (wie z. B. Gründerwettbewerbe) häufig die effizienteste Art der Förderung sind (Kambeck/Schmidt 2011: 383 ff.). Ähnliche Wettbewerbe in einzelnen Industriesparten (wie z. B. dem „BioRegio Contest") haben zu einer zufriedenstellenden Teilnahmebereitschaft unter den potenziellen Adressaten geführt (Dohse 2000: 1122). Aussichtsreiche Kooperationskonzepte erhalten in einem solchen Wettbewerb finanzielle Zuschüsse.

Von einem durchzuführenden „Wettbewerb um Kooperationen" dürften wirkungsvolle positive Anreize für eine Intensivierung von Kooperationsstrategien im Ruhrgebiet ausgehen. In Anlehnung an die in Kapitel 4 formulierten Anforderungen an Kooperationen müssten bei einem solchen Wettbewerb folgende Punkte in einem vorzulegenden Kooperationskonzept eindeutig formuliert sein: Erstens müssen die konkreten Verbesserungen, die mit der Kooperation erreicht werden sollen, explizit ausgearbeitet werden. Diese klaren Zielformulierungen verhindern Scheinkooperationen, die lediglich aus Prestigegründen eingegangen werden. Zweitens muss der Zeitrahmen bestimmt werden, in dem die Ziele der Kooperation erreicht werden sollen. Bei umfangreichen und lang andauernden Kooperationen sollten auch Zwischenziele zeitlich vorgegeben sein. Drittens müssen der erwartete Nutzen aus der Kooperation - nach Möglichkeit - beziffert und die Verteilung der Gewinne vorab festgelegt werden. Viertens müssen die geplanten

\footnotetext{
${ }^{10}$ Das Stärkungspaktgesetz Nordrhein-Westfalen, das hoch verschuldeten Kommunen finanzielle Hilfe gewährleistet, wenn diese eine strukturelle Haushaltssanierung in Angriff nehmen, funktioniert nach ähnlichem Prinzip. Allerdings stellt der Stärkungspakt an sich keinen - in diesem Absatz angesprochenen - negativen Anreiz dar, so dass er an dieser Stelle nicht weiter erörtert wird.
} 
Kooperationspartner klar benannt sein, wobei der spätere Beitritt von weiteren Partnern möglich sein sollte. Allerdings sollten gewisse Hürden für einen zwischenzeitlichen Ausstieg aufgebaut werden, wenn ein Ausstieg die Umsetzung der gesamten Kooperation gefährden kann. Fünftens muss das Konzept klar vorgeben, welche Kompetenzen an die Kooperation übertragen werden. Mit dieser Festlegung der Kompetenzen ist auch die Festlegung der Ausstiegskriterien gekoppelt. Und sechstens muss mit der Kooperation eine zwingende Evaluierbarkeit durch Dritte gegeben sein.

Die Teilnahme sollte vor allem auf kommunale Kooperationen ausgerichtet sein, wobei die Kooperationsthemen nicht eingeschränkt werden sollten. Neben den Kommunen selbst kommen auch Unternehmen in Frage, die als Partner die Erfüllung des Kooperationszweckes sinnvoll unterstützen können. Zwingende Voraussetzung sollte lediglich sein, dass einer der Kooperationsräume im Ruhrgebiet liegt, so dass auch sinnvolle Kooperationen, die über die Grenzen hinweg gehen, einbezogen werden. Die Bewertung der Konzepte sollte sich daran orientieren, ob erwartet werden kann, dass die Kooperation ein besonders hohes Einsparpotenzial hervorbringt, eine besondere Qualitätsverbesserung mit sich bringt oder besonders zukunftsweisende Investitionen gefördert werden.

Dabei stehen bei der konkreten Umsetzung des Wettbewerbs verschiedene Gestaltungsmöglichkeiten für den Gewinn zur Auswahl. Grundsätzlich kann zwischen drei zentralen Systemen unterschieden werden: Ein fixer Gewinn, eine anteilige Übernahme der Anfangskosten oder eine Bezuschussung der zu erwartenden Einsparungen bzw. Gewinne. Sowohl die anteilige Übernahme der Kosten als auch die anteilige Bezuschussung der Einsparpotenziale bergen die ,Gefahr', dass unkalkulierbar hohe Preisgelder ausbezahlt werden müssen. Dem kann durch eine Begrenzung der Fördergelder Rechnung getragen werden, wobei man wiederum die Auslobung zu geringer zusätzlicher Anreize vermeiden muss. Keines der Anreizsysteme ist somit problemlos. Eine anteilige Bezuschussung des entstandenen Nutzens durch die Kooperation hat aber - im Gegensatz zu den anderen Möglichkeiten - den Vorteil, dass für die Teilnehmer der Kooperation ein dauerhafter Anreiz bleibt, den Gewinn (Nutzen) der Kooperation zu maximieren.

Die Wettbewerbsform umgeht zwei zentrale Probleme bei der Findung eines optimalen Kooperationsraumes. Zum einen ist keine von oben bestimmende Instanz notwendig, um zunächst einen Kooperationsraum festzulegen. Dieser Raum kann entsprechend dem „bottom-up“-Gedanken wesentlich besser auf der unteren, die Sachlage vor Ort kennenden Ebene festgelegt werden. Zum anderen wird durch den Wettbewerb ein Anreiz gesetzt, der das kommunale Kirchturmdenken durchbrechen kann. Ein Wettbewerb stellt dabei nicht nur für den Gewinner eine Verbesserung der Situation dar. Die Aussicht auf den Gewinn des Wett- bewerbs schafft für alle Teilnehmer Anreize, ein Kooperationskonzept zu erarbeiten, das gegebenenfalls auch ohne eine Prämierung umgesetzt werden kann.

Wenn der Wettbewerb für Kooperationen erfolgreich gestaltet werden kann, dürften eine Vielzahl von Kooperationskonzepten voraussichtlich auch umgesetzt werden. Ein erfolgreicher Wettbewerb würde erstmals zu einer Offenlegung des Nutzens einer deutlichen Intensivierung von regionalen Kooperationen für Politik, Bürger und Kommunen führen. Zudem gibt er Leitlinien vor, die für bestehende und mögliche neue Kooperationen in der Kooperationslandschaft Ruhr Potenziale zur Verbesserung bieten. Der Politik dürfte es sehr schwer fallen, die dann absehbaren Verbesserungen aus Prestige- oder Machterhaltungsgründen nicht zu nutzen.

Danksagung Dieser Beitrag beruht auf dem RWI-Projektbericht (2011) „Den Wandel gestalten. Anreize für mehr Kooperation im Ruhrgebiet", durchgeführt im Auftrag der RAG-Stiftung. Wir bedanken uns bei Thomas K. Bauer, Rüdiger Budde, Sonja Kassenböhmer, Uwe Neumann, Colin Vance und Lina Zwick sowie zwei anonymen Gutachtern für ihre Kommentare und bei Andrea Cassel und Jenny Neumann für ihre Unterstützung beim Erstellen des Manuskripts.

\section{Literatur}

Benz, A.; Fürst, D. (2003): Erfolgsbedingungen für „,Regional Governance“ - Resümee. In: Adamaschek, B.; Pröhl, M (Hrsg.): Regionen erfolgreich steuern. Regional Governance - von der kommunalen zur regionalen Strategie. Gütersloh, 189-211.

Blatter, J.; Knieling, J. (2009): Metropolitan Governance - Institutionelle Strategien, Dilemmas, Variationsmöglichkeiten für die Steuerung von Metropolregionen. In: Knieling, J. (Hrsg.): Metropolregionen: Innovation, Wettbewerb, Handlungsfähigkeit. Hannover, 224-269. = Forschungs- und Sitzungsberichte der ARL, Band 231.

Blotevogel, H. H. (2001): 80 Jahre regionale Selbstverwaltung im Ruhrgebiet. In: Habrich, W.; Hoppe, W (Hrsg.): Strukturwandel im Ruhrgebiet: Perspektiven und Prozesse. Dortmund, 9-24. = Duisburger Geographische Arbeiten, Band 23.

Blotevogel, H. H. (2009): Raumwissenschaftliche Studie zur Gliederung des Landes Nordrhein-Westfalen in regionale Kooperationsräume. Fakultät Raumplanung, Technische Universität Dortmund. Dortmund.

Blume, T. (2009): Die ökonomischen Effekte regionaler Kooperation. Theorie und Empirie am Beispiel monozentrischer Regionen in Westdeutschland. Marburg.

Bogumil, J. (2011): Steuerung und Koordination der „Metropolregion“ Ruhrgebiet. In: Engel, K.; Großmann, J.; Hombach, B (Hrsg.): Phönix Flieg! Das Ruhrgebiet entdeckt sich neu. Essen, 577-593.

Danielzyk, R.; Kirchhof, R.; Knüpfer, U.; Laurin, S.; Münter, A.; Reicher, C.; Tenfelde, K.; Welt, J. (2010): Denkschrift der Bürgerschaftlichen Initiative RuhrStadt (herausgegeben von Pro Ruhrgebiet e. V. in Zusammenarbeit mit der Bürgerschaftlichen Initiative RuhrStadt). Essen.

Dohse, D. (2000): Technology policy and the regions: the case of the BioRegio contest. In: Research Policy 29, 9, 1111-1133.

Franz, P. (2011): Politische Institutionalisierung und Governance-Formen der deutschen Metropolregionen im Vergleich. In: Wirtschaft im Wandel 17, 11, 387-394. 
Frey, B. S.; Eichenberger, R. (2001): Metropolitan Governance for the Future: Functional Overlapping Competing Jurisdictions. In: Swiss Political Science Review 7, 3, 124-130.

Fürst, D. (2003): Steuerung auf regionaler Ebene versus Regional Governance. In: Informationen zur Raumentwicklung 8/9, 441-450.

Fürst, D. (2006): Regional Governance - Ein Überblick. In: Kleinfeld, R.; Plamper, H.; Huber, A. (Hrsg.): Regional Governance, Band 1. Göttingen, 37-60.

Healey, P. (2002): On Creating the ,City' als a Collective Resource. In: Urban Studies 39, 10, 1777-1792.

ILS (Institut für Landes- und Stadtentwicklungsforschung) (2009): Planung, Konzepte und Strategien der Stadt- und Regionalentwicklung im Ruhrgebiet. Expertise zu den Kooperationen im Ruhrgebiet. Dortmund.

Kambeck, R.; Schmidt, C. M. (2011): Den Strukturwandel richtig begleiten. In: Engel, K.; Großmann, J.; Hombach, B (Hrsg.): Phönix Flieg! Das Ruhrgebiet entdeckt sich neu. Essen, 367-387.

Knieling, J. (2003): Kooperative Regionalplanung und Regional Governance: Praxisbeispiele, Theoriebezüge und Perspektiven. In: Informationen zur Raumentwickung 8/9, 463-478.

Kübler, D. (2003): „Metropolitan Governance“ oder: Die unendliche Geschichte der Institutionenbildung in Stadtregionen. In: Informationen zur Raumentwicklung 8/9, 535-541.

Langhagen-Rohrbach, C.; Fischer, R. (2005): Regionalwerkstatt FrankfurtRheinMain. Region als Prozeß? In: Standort. Zeitschrift für Angewandte Geographie 29, 2, 76-80.
Laurin, S. (2010): Der große kleine Verband. In: Welt am Sonntag vom 3. Oktober 2010. Online unter: http://www.welt.de/die-welt/ regionales/koeln/article10039316/Der-grosse-kleine-Verband. html (letzter Zugriff am 18.01.2013).

Münter, A.; Prossek, A. (2011): Handlungsräume und Raumbilder. Räumliche Muster von Regionalisierungen im Ruhrgebiet. In: Reicher, C.; Kunzmann, K. R.; Polívka, J.; Roost, F.; Wegener, M.; Utku, Y (Hrsg.): Schichten einer Region. Kartenstücke zur räumlichen Struktur des Ruhrgebiets. Berlin, 182-215.

Reicher, C.; Kunzmann, K. R.; Polívka, J.; Roost, F.; Wegener, M.; Utku, Y. (Hrsg.) (2011): Schichten einer Region. Kartenstücke zur räumlichen Struktur des Ruhrgebiets. Berlin.

RVR (Regionalverband Ruhr) (2010): Bericht über die Beteiligungen des Regionalverbandes Ruhr für das Jahr 2009. Essen.

RWI (Rheinisch-Westfälisches Institut für Wirtschaftsforschung) (2011): Den Wandel gestalten. Anreize für mehr Kooperation im Ruhrgebiet. Studie im Auftrag der RAG-Stiftung. Essen.

Speer, A.; Denkel, M.; Winterhager, R.; Schotte, N.; Bergmann, H. (2009): Ruhrplan 21 - Wandel, Vielfalt, Fairness. Albert Speer und Partner GmbH. Frankfurt am Main.

Tiebout, C. (1956): A Pure Theory of Local Expenditures. In: The Journal of Political Economy 64, 5, 416-424.

Universitätsallianz Ruhr (2007): Kooperationsvereinbarung der Universitäten Duisburg-Essen, Bochum und Dortmund.

WMR (Wirtschaftsförderung metropoleruhr) (2008): Konzept Ruhr Strategie zur nachhaltigen Stadt- und Regionalentwicklung in der Metropole Ruhr. Mülheim an der Ruhr. 